\title{
Antibody Responses in Ostriches (Struthio camelus) Vaccinated with Commercial Live and Killed Newcastle Disease Vaccines
}

\author{
Kouji SAKAI ${ }^{1)}$, Genki SAKABE ${ }^{1)}$, Orie TANI ${ }^{1}$, Masayuki NAKAMURA ${ }^{1)}$ and Kazuaki TAKEHARA ${ }^{1)}$ \\ ${ }^{1)}$ Laboratory of Zoonoses, School of Veterinary Medicine and Animal Sciences, Kitasato University, Towada, Aomori 034-8628, Japan
}

(Received 2 September 2005/Accepted 6 February 2006)

\begin{abstract}
Three ostriches (Struthio camelus) were immunized with commercially available live and killed Newcastle disease (ND) vaccines for chickens and the antibody responses to the ND vaccines were evaluated by a virus-neutralization (VN) test. Primary vaccination with the live vaccine, B1, by eye drop was followed with two shots of alum-precipitated killed vaccine via subcutaneous injection in the neck. As a final booster, another live vaccine, Clone 30, was used by eye drop. A VN antibody titer, more than 1:10 was observed for 6 months. This is the first report on the use of a live vaccine by eye drop as a booster in ostriches as well as evaluating responses to ND vaccines using the $\mathrm{VN}$ test in this avian species.

KEY WORDS: Newcastle disease virus (NDV), ostrich (Struthio camelus), vaccination.
\end{abstract}

J. Vet. Med. Sci. 68(6): 627-629, 2006

Newcastle disease (ND) is the most important infectious disease in birds throughout the world. The causative agent of ND virus (NDV) is classified with the other eight avian paramyxoviruses in the genus Avulavirus, sub-family Paramyxovirinae, family Paramyxoviridae, order Mononegavirales [8]. Because of the severe nature of the disease and the associated consequences, ND is included as an Office International des Epizooties list A disease. NDV is spread on farms either by aerosol or by the fecal-oral route, whereas feed vehicles, personnel, etc., can act as mechanical carriers introducing the virus to uncontaminated areas [1]. In Japan ND outbreaks occurred in commercial poultry farms in the 1960s and live ND vaccines were subsequently introduced into the vaccination program. Following this vaccination regimen, minimal ND problems occurred for long periods, but in the late 1990s, sporadic ND outbreaks were again observed, causing serious damage to large scale commercial farms.

The isolation of NDV has previously been recorded in commercial ostriches in foreign countries $[4,5,10]$. In the countries that are leading producers and major exporters of ostrich products such as South Africa, it is important to reduce the risk of NDV transmission by effective vaccination. Several groups have reported the use of vaccine regimens in ostriches. Huchzermeyer and Gerdes [4] recommended the routine vaccination of ostriches with standard poultry vaccines using combinations of live and killed emulsified vaccines. A vaccination program for ostrich chicks with and without maternal antibodies has been suggested by Madeiros [6]. However, these authors did not determine the resulting immune responses to establish the efficacy of their vaccination programs. Cadman et al. [3] were the first to show that intramuscular vaccination of ostriches with an oil-emulsion (Texas GB strain) vaccine led to a serum antibody response.

In Japan commercial ostriches were introduced in 1988 and the ostrich numbers increased rapidly over the next 15 years, exceeding 9,000 birds by 2002. Since ostriches are kept mainly in a free-range situation, they easily come in contact with wild birds and animals that may be infected with pathogens including bacteria, viruses, and parasites. Because of this, there is increased impetus by ostrich producers in Japan to establish preventive medicine practices such as disease monitoring and surveillance. In our previous serological surveillance of NDV in Japan, 12.2\% of 181 slaughter-age ostriches contained NDV-specific neutralizing antibodies and the highest titer was recorded as high as 1:1,000 even without any vaccination history [9]. The high titer suggested that these ostriches might have been infected with virulent NDV. These data demonstrate that NDV has infiltrated into ostrich farms in Japan. Our ultimate goal is the development of a ND vaccination regimen in ostriches to obtain the desired immunity and protection against ND. Therefore, the objective of this study was to determine antibody responses in ostriches vaccinated with commercially available live and killed ND vaccines for chickens.

Three ostrich chicks at 13-week-old were obtained from Yamagata-Asahi Ostrich Industry Center Co. in Yamagata prefecture and these birds were reared in a disease-free building in Kitasato University with the appropriate feeding and husbandry. The experiment started at 14 weeks of age.

For immunization with the ND vaccines, two live vaccines, ND B1 (The Chemo-Sero-Therapeutic Research Institute, Kumamoto, Japan) and Clone 30 (Intervet International B. V., The Netherlands) and one inactivated vaccine $\mathrm{NB}$, a combined vaccine of alum-precipitated NDV strain Sato and infectious bronchitis virus strain Ishida (Nippon Institute for Biological Science, Tokyo, Japan) were used. At 14 weeks of age, all ostriches were vaccinated with the live $\mathrm{B} 1$ vaccine by eye drop ( $5 \mathrm{X}$ the chicken dose rate). At 4 weeks and 12 weeks after the first vaccination, the inactivated NB vaccine was administered as subcutaneous injection in the neck ( $2 \mathrm{X}$ and $4 \mathrm{X}$ the chicken dose rate, respectively). As the final booster, they were vaccinated 


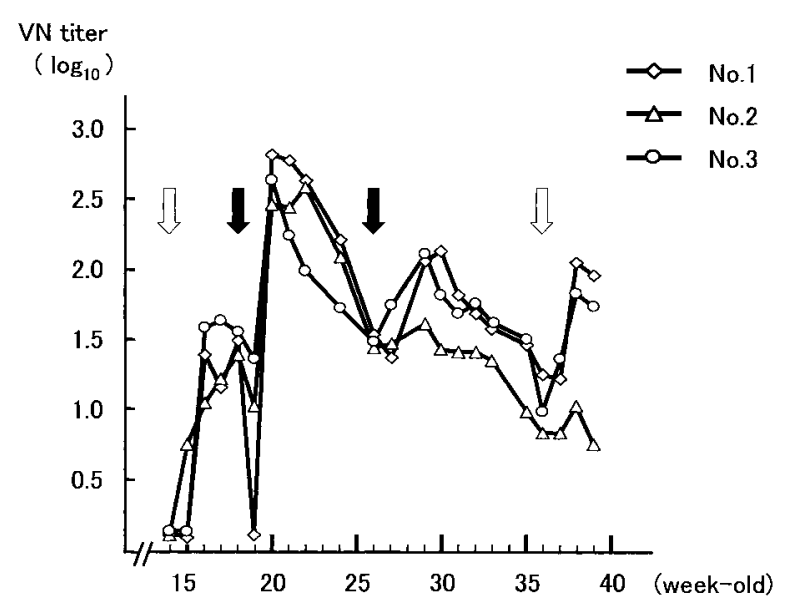

Fig. 1. VN titers following vaccination with live and killed ND Vaccinations were indicated with arrows ( : the live vaccine, $\downarrow$ : the inactivated vaccine).

with the live Clone 30 vaccine by eye drop ( $5 \mathrm{X}$ the chicken dose rate) at 22 weeks after the first vaccination. This immunization was performed with a modification of the ND vaccination schedule for ostriches by Madeiros [6]. Daily clinical monitoring of the ostriches was carried out for the duration of the experiment. Blood was collected from the jugular vein at indicated time as described in Fig. 1 after the first vaccination. The blood was centrifuged and the resultant serum was harvested and frozen at $-20^{\circ} \mathrm{C}$ until tested. No clinical signs of diseases were observed in any of the birds tested for the duration of the experiment.

Chicken embryo fibroblasts were prepared from 10-dayold chicken embryos as described $[11,12]$. A virus-neutralization (VN) test for NDV was performed by the plaquereduction method with a constant amount of virus and varying serum dilution. Sample sera and antiserum against NDV strain Ishii [13] were serially four-fold diluted, and mixed with equal volumes of NDV strain Sato. The neutralizing antibody titer was calculated at $50 \%$ plaque reduction point by the Behrens-Kaerber's method [7].

Antibody responses of the VN titer to the vaccination with ND vaccines are shown in Fig. 1. The VN antibody titers of the three ostriches began to increase and reached maximum titers $(1: 31,1: 25$, and $1: 43$, respectively) at 3-4 weeks after the first vaccination. The titers decreased slightly the first week after the second vaccination but at 2 weeks, the titers surpassed those observed following the first vaccination, confirming a booster effect $(1: 429,1: 381$, and 1:594, respectively). The birds maintained high VN antibody titer (more than 1:100) even at 4 weeks after the second vaccination. The third vaccination with the inactivated ND vaccine at 13 weeks after primary vaccination could not induce titers to the levels of the second vaccination but could induce VN antibody titers (more than 1:10) that lasted for another 8 weeks. After the fourth and final vaccination with the live ND vaccine, Clone 30 , elevated antibody titers were again induced and maintained for 3 weeks the experiment was terminated (Fig. 1).

As far as we could determine, there is no detailed data with respect to monitoring the antibody response in ostriches using the VN test, however, the VN test would be the most accurate and most reliable. Therefore, we used the $\mathrm{VN}$ test for the detection of anti-NDV antibodies in ostrich serum.

In the present study, we did not administer the live vaccine by drinking water, because this route seemed to be inadequate to raise antibody response. When we tested ostrich sera immunized 3 times at 2 weeks interval with live ND vaccine by drinking water in a prefecture livestock hygiene service center, only 9 of 30 ostrich sera were positive with VN test. Therefore, we considered that VN antibodies could not be properly induced by drinking water vaccination. Since the growth rate of ostrich chicks less than 3-month-old is very low, the experiment started at 13week-old in the present study. Huchzermeyer and Gerdes [4] and Madeiros [6] advocated the routine use of a live vaccine (6X the chicken dose rate) for immunization of ostrich chicks by eye drop at early age. On the other hand, Blignaut et al. [2] reported that immunization by eye drop with a live La Sota vaccine (one drop in each eye, $10^{6}$ mean embryo infective dose) for 5-week-old ostriches did not elicit humoral immune responses that could be detected by an enzyme-linked immunosorbent assay. The reason for this was not given. In the present study, the live ND B1 vaccine administered by eye drop ( $5 \mathrm{X}$ the chicken dose rate) could induce antibodies in 14-week-old ostrich chicks.

An inactivated alum-precipitated vaccine we used in this study induced further immune responses and the antibody titer was maintained for a long period. No inflammatory side reaction was detected at the injection site of the inactivated vaccine. These results agree with the results of Blignaut et al. [2] with the inactivated alum-precipitated La Sota vaccine. South Africa Export and Source Agents have advocated that oil vaccine induces inflammatory reactions at the vaccination site.

We also used the live vaccine, Clone 30 by eye drop as a booster. Madeiros [6] reported that Clone 30 stimulated a good antibody response. This vaccine could raise antibody titer even in the immunized ostriches that had detectable antibodies and the titer was maintained until the experiment had finished. Because intramuscular and subcutaneous injection of adult ostriches with inactivated vaccine is very laborious and dangerous, the booster by eye drop may be useful for labor-saving and safety. Unfortunately we did not check whether Clone 30 virus was isolated after immunization. It remains to be determined whether live ND vaccine viruses will persist in ostriches.

In conclusion, our study has shown that commercially available live and killed ND vaccines for chickens induce good antibody response in ostriches. After the first immunization, the birds maintained the VN antibody titer (approximately more than 1:10). In a previous study [9], we demonstrated that NDV had infiltrated into ostrich farms 
indicating the importance of establishing an ND vaccination program for ostriches.

ACKNOWLEDGMENT. This work was supported in part by a Grant for Scientific Research (No. 2840) from Kitasato University, School of Veterinary Medicine and Animal Sciences.

\section{REFERENCES}

1. Alexander, D. J. 1995. J. Comp. Pathol. 112: 105-126.

2. Blignaut, A., Burger, W. P., Morley, A. J. and Bellstedt, D. U. 2000. Avian Dis. 44: 390-398.

3. Cadman, H. F., Kelly, P. J., N. D. De Angelis, Rohde, C., Collins, N. and Zulu, T. 1997. Avian Pathol. 26: 357-363.

4. Huchzermeyer, F.W. and Gerdes, G. H. 1993. J. S. Afr. Vet. Assoc. 64: 140.

5. Jorgensen, P. H., Handberg, K. J., Lomniczi, B., Ruth, J., Man- vell, R. J., Holm, E. and Alexander, D. J. 1998. Avian Pathol. 27: $352-358$.

6. Madeiros, C. 1997. Vet. Rec. 140: 188.

7. Matumoto, M. 1949. Jpn. J. Exp. Med. 20: 175-179.

8. Mayo, M. A. 2002. Arch. Virol. 147: 1655-1663.

9. Sakai, K., Yada, K., Sakabe, G., Tani, O., Miyaji, K. Nakamura, M. and Takehara, K. 2006. J. Vet. Med. Sci. 68: 491494.

10. Samberg, Y., Hadash, D. U., Perelman, B. and Meroz, M. 1989. Avian Pathol. 18: 221-226.

11. Takehara, K., Kiuchi, H., Kuwahara, M., Yanagisawa, F., Mizukami, M., Matsuda, H. and Yoshimura, M. 1991. J. Vet. Med. Sci. 53: 479-486.

12. Takehara, K., Shinomiya, T., Kobayashi, H., Azuma, Y., Yamagami, T. and Yoshimura, M. 1987. Avian Dis. 31: 125129.

13. Yachida, S., Iritani, Y. and Katagiri, K. 1979. Avian Dis. 23: 979-982. 